09,12

\title{
Двумерные световые пули в среде с неоднородной плотностью углеродных нанотрубок
}

\author{
(C) И.С. Двужилов ${ }^{1}$, М.Б. Белоненко ${ }^{1,2}$ \\ ${ }^{1}$ Волгоградский государственный университет, \\ Волгоград, Россия \\ ${ }^{2}$ Волгоградский институт бизнеса, \\ Волгоград, Россия \\ E-mail: dvuzhilov.ilya@gmail.com
}

(Поступила в Редакцию 25 января 2016 г.

В окончательной редакции 12 июля 2017 г.)

Рассмотрена теоретическая задача о динамике распространения предельно коротких оптических импульсов (световых пуль) в среде с неоднородной плотностью углеродных нанотрубок. Показано, что световые пули распространяются стабильно и устойчиво.

DOI: $10.21883 /$ FTT.2018.02.45392.025

\section{1. Введение}

Двумерные предельно короткие оптические импульсы (световые пули) привлекают наибольшее внимание среди нелинейных оптических явлений. Не стоит также забывать о важности практических приложений изучения их динамики в системах оптических вычислений [1].

В линейных средах световые пули неустойчивы вследствие их уширения в силу дисперсии. В свою очередь, для стабильного существования световых пуль требуется нелинейная среда, в которой эффекты, связанные с дисперсией, восполнялись бы нелинейными эффектами [2-4].

В последнее время в качестве такой среды рассматриваются углеродные нанотрубками (УНТ), являющиеся уникальным строительным блоком в современной наноэлектронике и также средой для образования световых пуль, в которой нелинейность обусловлена непараболичностью закона дисперсии электронов в нанотрубках и их взаимодействием с полем светового импульса [5-7]. Подобной теме посвящено много работ [8-10], но проблема состоит в том, что в однородной среде углеродных нанотрубок скорость распространения световых пуль определяется исключительно показателем преломления среды, и она не может быть изменена в довольно широких пределах. Решением такого рода проблемы может послужить модуляция показателя преломления среды, тем самым сформировав так называемую неоднородную среду. В этом случае удастся управлять скоростью световой пули в среде такого рода. [11-13].

В большинстве работ рассматривается среда, содержащая УНТ и модулированный показатель преломления одновременно, что в свою очередь вызывает некоторые практические трудности в создании таких сред. Оптимальным является способ получения сред, в которых распределение УНТ неоднородное, что приводит к возникновению пространственной модуляции показателя преломления, а вместе с этим и изменению скорости распространения импульсов, которая позволит управлять временем задержки.

Важность практического применения, а также приведенные выше соображения и послужили стимулом для написания данной статьи.

\section{2. Постановка задачи}

В рассматриваемой задаче будут использоваться следующие основные приближения. Во-первых, не учитывается дифракционное расплывание лазерного пучка в направлении вдоль оси УНТ. Строго говоря, это является не приближением, а требованием, чтобы волновой фронт импульса в заданном направлении был плоским. Иными словами, это требование к характерному масштабу неоднородности вдоль оси УНТ. Так, если мы рассматриваем задачу на временах порядка 10 ps, то характерная длина пробега импульса будет порядка $3 \mathrm{~mm}$. Из соотношения для дифракционной длины получаем что характерный масштаб неоднородности должен быть больше $0.1 \mathrm{~mm}$, что легко достижимо на практике. Оси нанотрубок считаем параллельными друг другу и параллельными оси $z$. Во-вторых, не учитывается электрическое поле подложки. Отметим, что поскольку типичный размер УНТ и расстояние между ними много меньше, чем типичный размер пространственной области, в которой локализован предельно короткий импульс, можно использовать приближение сплошной среды и считать ток распределенным по объему. Будем везде далее полагать, что электрическое поле световой пули имеет вид $\mathbf{E}(\mathbf{r})=e_{z} E_{z}(x, y)$. УНТ рассмотрим как однослойный графеновый лист скрученный в цилиндр, ограничиваясь учетом только $\pi$-электронов, предполагая, что их движение может быть описано в приближении сильной связи. Радиус УНТ считается много меньшим по сравнению с характерным размером световой пули, что позволяет пренебречь пространственной неоднородностью поля в трубках. 

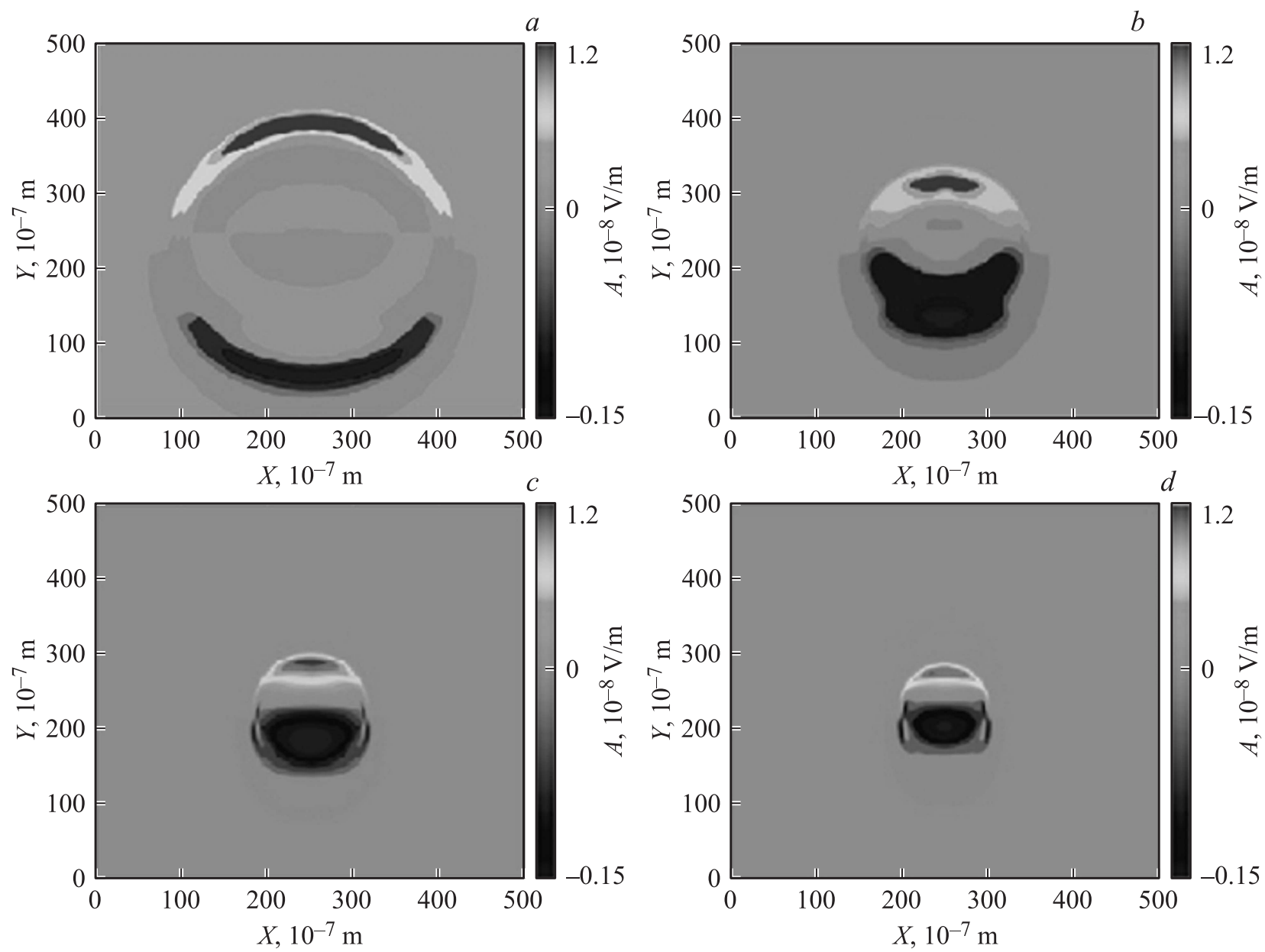

Рис. 1. Распространение световой пули в среде с неоднородной плотностью (период решетки $\chi=0.125 \mu \mathrm{m})$ углеродных нанотрубок в различные моменты времени $\left.\left.\left.T: a) 16 \cdot 10^{-12} \mathrm{~s}, b\right) 10 \cdot 10^{-12} \mathrm{~s}, c\right) 6 \cdot 10^{-12} \mathrm{~s}, d\right) 5 \cdot 10^{-12} \mathrm{~s}$. По осям отложены относительные единицы координат и электрического поля.

В одноэлектронном приближении уравнение Шредингера принимает следующий вид [14]:

$$
i \hbar \frac{\partial \psi(t, r)}{\partial t}=-\frac{\hbar^{2}}{2 m_{0}} \Delta \psi(t, r)+[W(r)-e(E \cdot r)] \psi(t, r),
$$

где $W(r)$ - потенциал, создаваемый атомами.

Стационарное решение уравнения (1) может быть представлено в виде:

$$
\psi=\sum_{l, p} C_{l}(p) \psi_{l}(p, r)
$$

где $l$ - набор квантовых чисел, характеризующих состояния $\pi$-электронов с заданным квазиимпульсом. $\Psi_{l}(\mathbf{p}, \mathbf{r})=\hbar^{-0.5} \exp (i \mathbf{p r} / \hbar) u_{l, \mathbf{p}}(\mathbf{r})-$ функции Блоха $\mathrm{c}$ амплитудой $u_{l, \mathbf{p}}$ периодичной к произвольному вектору решетки $\mathbf{a}: u_{l, \mathbf{p}}(\mathbf{r}+\mathbf{a})=u_{l, \mathbf{p}}(\mathbf{r})$. Здесь $\mathbf{a}=n_{1} \mathbf{a}_{1}+n_{2} \mathbf{a}_{2}$, $n_{1}, n_{2}$ - целые числа, $\mathbf{a}_{1}, \mathbf{a}_{2}$ - элементарные векторы гексагональной решетки графена. Здесь мы привели (2) для прояснения базиса, в котором будет решаться уравнение (1).
С учетом спектра нанотрубок [15], из уравнений (1) и (2) следует

$$
\hat{H}_{0}=\left(\begin{array}{cc}
0 & H_{12}(p) \\
H_{12}^{*}(p) & 0
\end{array}\right),
$$

что можно переписать в приближении сильной связи как

$$
H_{12}(p)=-\gamma_{0} \sum_{j=1}^{3} \exp \left(\frac{i p \tau_{i}}{\hbar}\right),
$$

где $\gamma_{0}-$ интеграл перескока $(2.7 \mathrm{eV}), \boldsymbol{\tau}_{i}-$ вектор, соединяющий атом с ближайшими соседями.

Тогда для матрицы плотности получаем следующие уравнения:

$$
\begin{gathered}
\frac{\partial \rho}{\partial t}+e E_{z} \frac{\partial \rho}{\partial p_{z}}=2 \frac{e}{\hbar} E_{z} R_{a b} \Phi, \\
\frac{\partial F}{\partial t}+e E_{z} \frac{\partial F}{\partial p_{z}}=\omega \Phi \\
\frac{\partial \Phi}{\partial t}+e E_{z} \frac{\partial \Phi}{\partial p_{z}}=\frac{e}{\hbar} E_{z} R_{a b}(2 \rho-1)-\omega F
\end{gathered}
$$



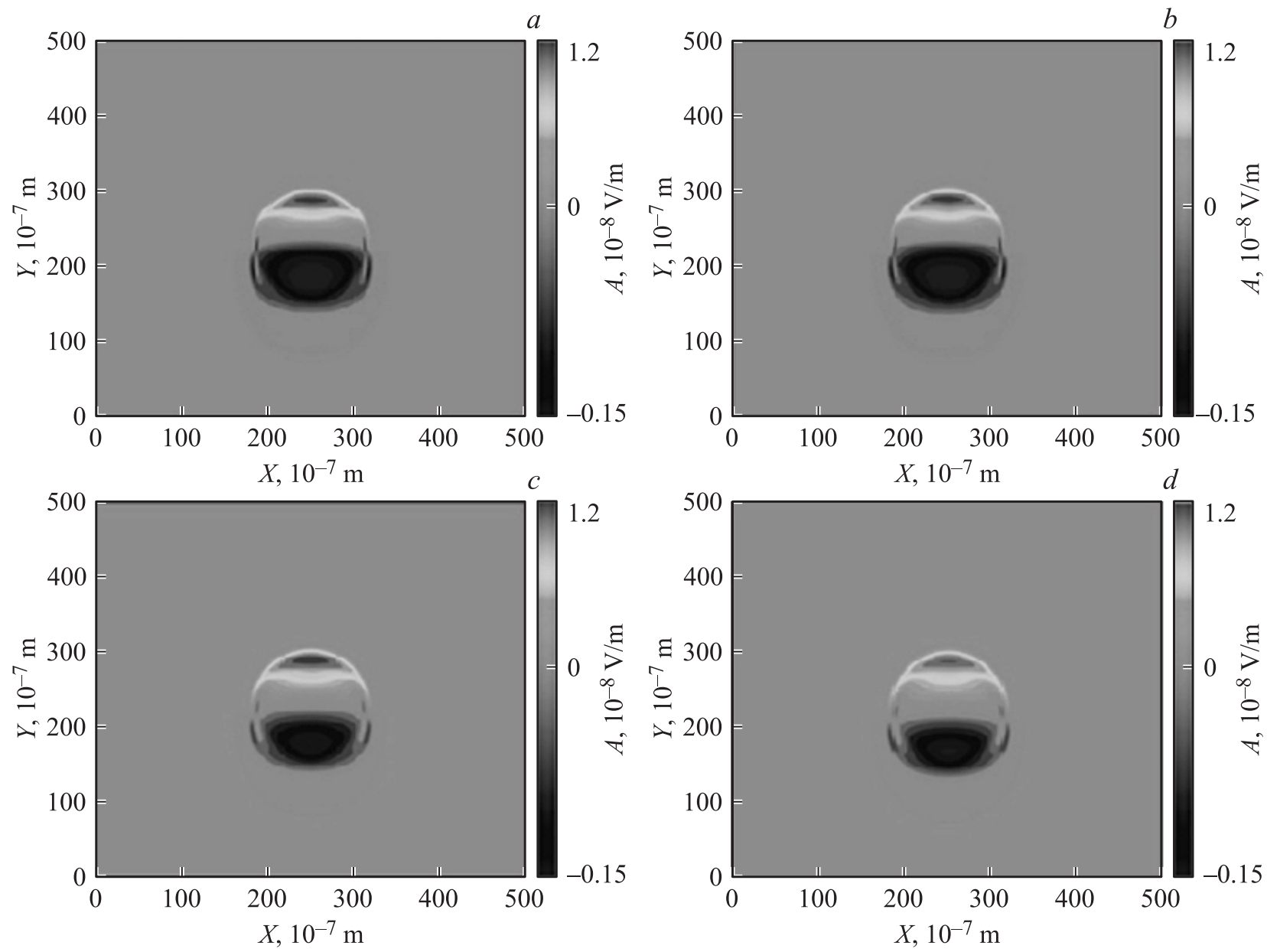

Рис. 2. Изменение формы импульса в среде с неоднородной плотностью углеродных нанотрубок (период решетки $\chi=0.125 \mu \mathrm{m})$ в фиксированный момент времени $T=6 \mathrm{ps}$ с различной глубиной модуляции $\mathrm{alf}_{0}=0.25: a$ ) 0.3alf $\left.\left.\left.\mathrm{al}_{0}, b\right) 0.2 \mathrm{alf}_{0}, c\right) 0.1 \mathrm{alf}_{0}, d\right) 0.05 \mathrm{alf}_{0}$. По осям отложены относительные единицы координат и электрического поля.

Для удобства численного интегрирования введем замену: $F=\operatorname{Re}\left(\rho_{c v}\right) ; \Phi=\operatorname{Im}\left(\rho_{c v}\right) ; \rho=\rho_{c v}-$ матрица плотности, $\omega-$ частота межзонного перехода, $e-$ электрический заряд. Индексы $c$ и $v$ относятся к зоне проводимости и к валентной зоне соответственно. Матрица плотности построена на волновых функциях зоны проводимости и валентной зоны

$$
R_{l l^{\prime} p}=\frac{i \hbar}{2} \int_{\Omega}^{0}\left(u_{l, p}^{*} \frac{\partial u_{l^{\prime}, p}}{\partial p_{z}}-\frac{\partial u_{l^{\prime}, p}}{\partial p_{z}} u_{l, p}^{*}\right) d^{2} r .
$$

Интегрирование ведется по объему $\Omega$ двумерной элементарной ячейки, и постоянная Планка положена равной единице.

Начальные условия задачи будут следующими:

$$
\rho_{t=0}=F_{0}\left(\varepsilon_{c}\left(p_{z}, s\right)\right) ;\left.F\right|_{t=0}=\left.\Phi\right|_{t=0}=0 .
$$

Это отражает тот факт, что при комнатной температуре электроны распределены согласно равновесному распределению Ферми с нулевым химическим потенциалом $(\mu=0)$. Граничные условия отражают периодичность решения в пространстве квазиимпульса (аналогично для $F$ и $\Phi)$

$$
\rho\left(t, \frac{\sqrt{3} \pi}{\omega_{c n} a}\right)=\rho\left(t,-\frac{\sqrt{3} \pi}{\omega_{c n} a}\right) .
$$

Квантово-механический оператор плотности тока может быть записан в виде

$$
\hat{j}_{z}(r)=-\frac{i e \hbar}{2 m_{0}}\left(\frac{\partial}{\partial z^{\prime}} \delta\left(r-r^{\prime}\right)+\delta\left(r-r^{\prime}\right) \frac{\partial}{\partial z^{\prime}}\right) .
$$

Разложим общую плотность тока на две составляющие: $\bar{j}_{z}=j_{1}+j_{2}$, где за внутризонные переходы отвечает

$$
j_{1}=\frac{4 e}{(2 \pi \hbar)^{2}} \int_{1 Z B} \frac{\partial s_{c}(p)}{\partial p_{z}} \rho(t, p) d^{2} p,
$$

а за межзонные переходы -

$$
j_{2}=\frac{8 e}{(2 \pi \hbar)^{2} \hbar} \int_{1 Z B} \varepsilon_{c}(p) R_{c v}\left(p_{z}, s\right) \Phi(t, p) d^{2} p .
$$

Здесь учтено, что $\rho_{v v}+\rho_{c c}=1$ и $\varepsilon_{v}=-\varepsilon_{c}$. 

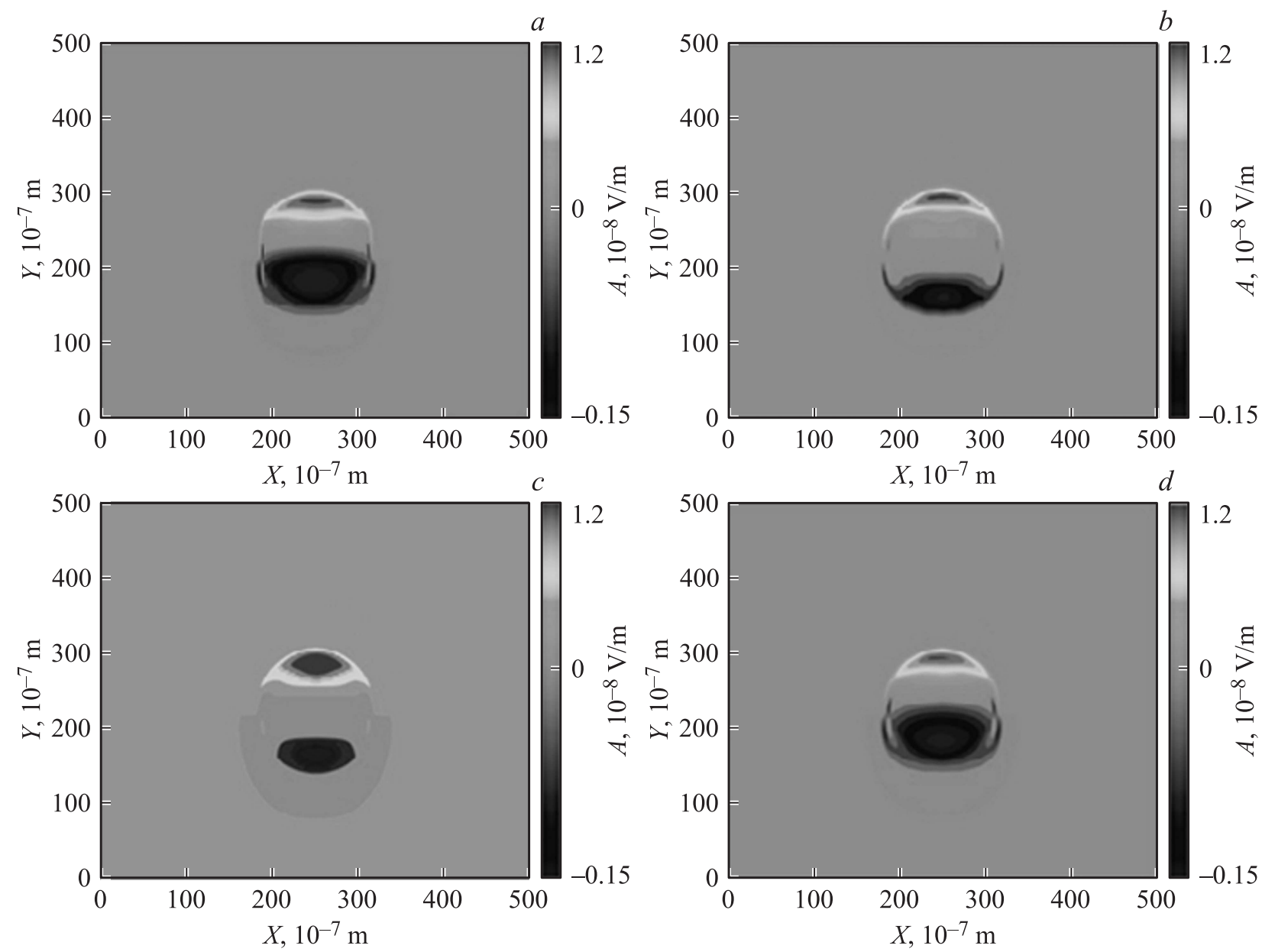

Рис. 3. Распространение световой пули в среде с неоднородной плотностью углеродных нанотрубок в фиксированный момент времени $T=6$ ps с различными периоднами решеток $\chi: a-0.125 \mu m, b-0.1 \mu m, c-0.25 \mu m, d-0.05 \mu m$. По осям отложены относительные единицы координат и электрического поля.

Запишем электрическое поле в калибровке $E=-1 \partial \mathbf{A} / c \partial t$ (где $c-$ скорость света в вакууме) и перейдем к обобщенному импульсу: $p \rightarrow p-e \mathbf{A} / c$, окончательно уравнения (5) приобретают вид, с учетом замены $\rho-0.5 \rightarrow \rho$

$$
\left\{\begin{array}{l}
\rho_{t}=-\frac{2}{c} A_{t} R_{c v} \Phi \\
F_{t}=\omega \Phi \\
\Phi_{t}=-\frac{2}{c} A_{t} R_{c v} \rho-\omega F .
\end{array}\right.
$$

Уравнения Максвелла для случая немагнитных диэлектрических сред приводятся к виду [15]

$$
\frac{\partial^{2} \mathbf{A}}{\partial x^{2}}+\frac{\partial^{2} \mathbf{A}}{\partial y^{2}}+\frac{4 \pi}{c} n(x, y)\left(j_{1}+j_{2}\right)-\frac{1}{c^{2}} \frac{\partial^{2} \mathbf{A}}{\partial t^{2}}=0
$$

где $A-$ вектор-потенциал, $t-$ время, $c-$ скорость света в вакууме. В (12) феноменологически введен коэффициент, который учитывает распределение УНТ в пространстве $n(x, y)$. В дальнейшем, в численных расчетах это распределение будет задано в виде $n c(x, y)=1+\alpha \cos (g x) \cos (f y)$, где $\alpha$ задает глубину модуляции нелинейности, а $g$ и $f$ - период модуляции. Отметим, что в данной работе рассматривается модуляция во всех направлениях.

Закон дисперсии УНТ типа zig-zag далее будет выбран в виде

$$
\varepsilon_{s}(p)= \pm \gamma \sqrt{1+4 \cos (a p) \cos (\pi s / m)+4 \cos ^{2}(\pi s / m)}
$$

где $s=1,2, \ldots, m$, нанотрубка имеет тип $(m, 0)$, $\gamma \approx 2.7 \mathrm{eV}, a=3 b / 2 \hbar, b=0.142 \mathrm{~nm}$ расстояние между соседними атомами углерода.

\section{3. Численный расчет}

Исследуемое уравнение (12) с учетом (11) и (9) решалось численно. Межзонный ток полагался равным нулю, т.е. считалось, что спектр световой пули лежит выше видимой части спектра, а максимальная частота колебаний в спектре пули - в ближней инфракрасной области. 

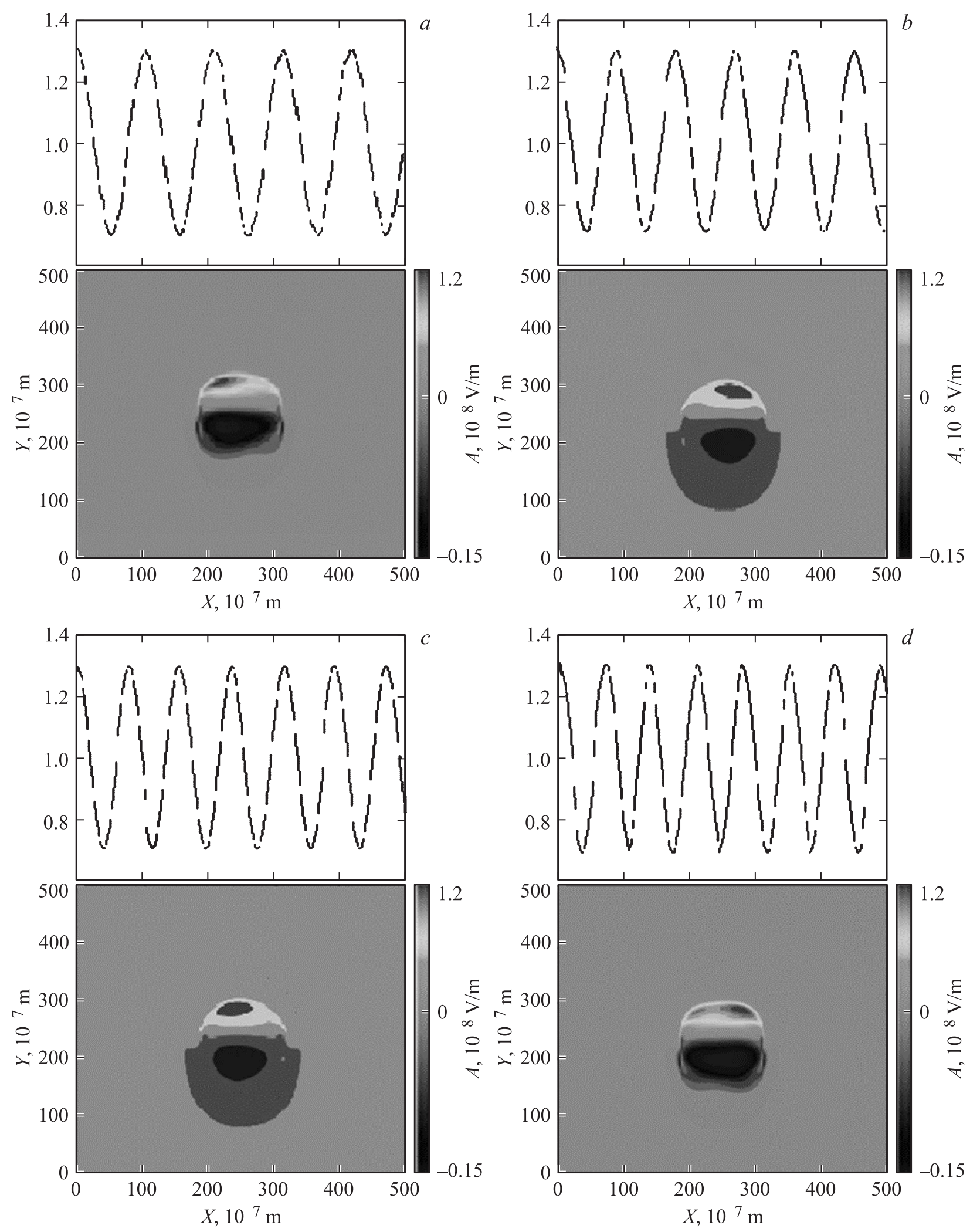

Рис. 4. Распространение световой пули в среде с неоднородной плотностью (период решетки $\chi=0.125 \mu \mathrm{m})$ углеродных нанотрубок в фиксированный момент времени $T=6 \mathrm{ps}$ с различными показателями модуляции плотности: $a) 0.06, b) 0.07, c) 0.08$, d) 0.09. По осям отложены относительные единицы координат и электрического поля. Вверху показано относительное изменение концентрации УНТ в поперечном направлении. 
Начальное условие для вектор-потенциала электрического поля световой пули выбиралось в гауссовой форме

$$
\begin{gathered}
\frac{d A(x, y, 0)}{d t}=\frac{2 Q x v}{\gamma^{2}} \exp \left(-\frac{\left(x-x_{0}\right)^{2}}{\gamma_{x}}\right) \exp \left(-\frac{\left(y-y_{0}\right)^{2}}{\gamma_{y}}\right) \\
A(x, y, 0)=Q \exp \left(-\frac{\left(x-x_{0}\right)^{2}}{\gamma_{x}}\right) \exp \left(-\frac{\left(y-y_{0}\right)^{2}}{\gamma_{y}}\right) .
\end{gathered}
$$

Здесь $Q$ - амплитуда импульса; $\gamma_{x}, \gamma_{y}$ - ширины импульса в направлении $x$ и $y$ соответственно; $v-$ начальная скорость импульса. Эти начальные условия соотвествуют распространению импульса вдоль оси $x$. Уравнение (12) решалось при помощи разностной схемы типа „крест“ [16]. Шаги по времени и координате определялись из стандартных условий устойчивости и уменьшались до тех пор, пока решение не изменялось в восьмом значащем знаке.

Как показали результаты численных расчетов, распространение световой пули является устойчивым и получившаяся эволюция представлена на рис. 1 .

Подобный характер распространения двумерного предельно короткого оптического импульса позволяет сделать вывод об устойчивом характере его динамики в среде с периодически меняющейся концентрацией УНТ. Отметим, что под световой пулей мы понимаем тут область максимальной интенсивности. Поскольку импульс в начальный момент имеет гауссову форму, его „края“ имеют малую интенсивность и распространяются в линейном режиме. Отличий при этом от линейного случая нет. В тоже время область с высокой интенсивностью попадает в нелинейный режим, когда и происходит „подавление“ дифракции, и основную роль играют нелинейные эффекты. Также на основании приведенного рис. 1 можно сказать, что среда с периодически меняющейся концентрацией УНТ замедляет распространение световой пули, как и предсказано теорией. Это связано (как и в случае брэгговских сред) с процессами отражения импульса от областей с разной концентрацией УНТ (а значит и разной диэлектрической проницаемостью) и дальнейшей интерференцией отраженных и прошедших импульсов.

Как видно из приведенных зависимостей, решение для двумерной световой пули в рассматриваемой среде остается локализованным, но меняет, вследствие поперечной дисперсии, свою пространственную структуру. Совместное действие эффектов расплывания импульса вследствие дисперсии и нелинейности приводят к образованию поперечной структуры, которая тем не менее остается локализованной в ограниченной пространственной области.

Как и следовало ожидать характер динамики импульса зависит от того, насколько сильно мы меняем концентрацию УНТ в среде, что представлено параметром $\alpha$ и приведено на рис. 2.

Зависимость же от периода модуляции концентрации УНТ представлена на рис. 3 и тем самым показано, что импульс несет в себе информацию о состоянии среды.
Важным результатом, полученным при исследовании зависимости динамики световой пули от модуляции плотности УНТ в поперечном направлении распространения, является тот факт, что световая пуля втягивается в область с большей концентрацией УНТ, что и представлено на рис. 4.

Это имеет достаточно простое объяснение. В области c большей концентрацией УНТ больше и величина эффективной нелинейности, а потому и выше интенсивность световой пули. Вместе с тем это позволяет корректировать траекторию световой пули путем создания волноводов из областей с большей концентрацией УНТ.

\section{4. Заключение}

Из проведенного исследования можно сделать следующие выводы:

1. Распространение предельно короткого оптического импульса устойчиво в неоднородной среде с углеродными нанотрубками. Как и следовало ожидать, неоднородность среды в значительной степени влияет на форму импульса, сглаживая его.

2. Установлено, что период неоднородности среды влияет на скорость распространения предельно короткого импульса. Увеличение периода приводит к тому, что импульс реже „отражается“ от „границ“ неоднородности, вследствие чего увеличивается его скорость. Таким образом, меняя период неоднородности, можно контролировать скорость распространения импульса, что важно для решения прикладных задач оптики.

3. Замедление импульса, а также изменение его формы происходит при увеличении глубины модуляции неоднородности, по причине сильной интерференции. Особенно сильные изменения формы наблюдаются на спаде предельно короткого оптического импульса.

\section{Список литературы}

[1] P.M. Goorjian. US Patent N 5,651,079 (22 July 1997).

[2] A.E. Kaplan, P.L. Shkolnikov Phys. Rev. Lett. 75, 2316 (1995).

[3] L.W. Casperson Phys. Rev. A 57, 609 (1998).

[4] T. Brabec, F. Krausz. Rev. Mod. Phys. 72, 545 (2000).

[5] T. Schafer, C.E. Wyane. Phys. D 196, 90 (2004).

[6] R. Saito, M.S. Dresselhaus, G. Dresselhaus. Physical properties of carbon nanotubes. Imperial College Press, London (1999). $251 \mathrm{p}$.

[7] S. Reich, C. Thomsen, J. Maultzsch. Carbon nanotubes. Basic concepts and physical properties. Wiley-VCH Verlag, Berlin. (2003). 218 p.

[8] P.J.F. Harris. Carbon nanotubes and related structures: New materials for the 21st century. Cambridge University Press, UK (2009). 299 p.

[9] S.A. Maksimenko, G.Ya. Slepyan. Nanoelectromagnetics of low-dimentional structure. In Handbook of nanotechnology. Nanometer structure: theory, modeling, and simulation. SPIE press, Bellingham (2004). 145 p. 
[10] H. Leblond, D. Mihalache. Phys. Rev. A 86, 043832 (2012).

[11] A.V. Zhukov, R.E. Bouffanais, G. Fedorov, M.B. Belonenko. J. Appl. Phys. 114, 143106 (2013).

[12] М.Б. Белоненко, Ю.В. Невзорова. Изв. РАН. Сер. физ. 78, 1619 (2014).

[13] M.B. Belonenko, Yu.V. Nevzorova, E.N. Galkina. Mod. Phys. Let. B 29, 1550041 (2015).

[14] A.V. Zhukov, R. Bouffanais, M.B. Belonenko, N.N. Konobeeva, Yu.V. Nevzorova, T.F. George. Eur. Phys. J. D 69, (2015).

[15] Н.Н. Якушкина, М.Б. Белоненко. ЖТФ 83, 155 (2013).

[16] Н.С. Бахвалов. Численные методы (анализ, алгебра, обыкновенные дифференциальные уравнения). Наука, М. (1975). 632 c. 